\title{
The effective design of bean bag as a vibroimpact damper
}

\author{
A.Q. Liu $^{\mathrm{a}, *}$, B. Wang ${ }^{\mathrm{b}}$, Y.S. $\mathrm{Choo}^{\mathrm{c}}$ and \\ K.S. Ong \\ ${ }^{a}$ School of Electrical \& Electronic Engineering, \\ Singapore \\ ${ }^{\mathrm{b}}$ School of Mechanical and Production Engineering, \\ Nanyang Technological University, Nanyang Avenue, \\ Singapore 639798, Singapore \\ ${ }^{\mathrm{c}}$ Department of Civil Engineering, National \\ University of Singapore, Singapore 117602, Singapore \\ ${ }^{\mathrm{d}}$ DSO National Laboratory, 20 Science Park Drive, \\ Singapore 118230, Singapore \\ Received 8 February 1996 \\ Revised 30 December 1999
}

The technique of a bean bag damper has been effectively applied in many engineering fields to control the vibroimpact of a structural system. In this study, the basic parameters responsible for the design of an effective bean bag: the size of beans, the mass ratio of the bean bag to the structure to which it is attached, the clearance distance and the position of the bag, are studied by both theoretical and experimental analyses. These will provide a better understanding of the performance of the bean bag for optimisation of damper design. It was found that reducing the size of beans would increase the exchange of momentum in the system due to the increase in the effective contact areas. Within the range of mass ratios studied, the damping performance of the damper was found to improve with higher mass ratios. There was an optimum clearance for any specific damper whereby the maximum attenuation could be achieved. The position of the bag with respect to nodes and antipodes of the primary structure determined the magnitude of attenuation attainable. Furthermore, the limitations of bean bags have been identified and a general criteria for the design of a bean bag damper has been formulated based on the study undertaken. It was shown that an appropriately configured bean bag damper was capable of reducing the amplitude of vibration by $80 \%$ to $90 \%$.

Keywords: Bean bag, vibroimpact, shock, vibration and impact damper

${ }^{*}$ Corresponding author; Tel.: +65 790 4336; Fax; +65 793 3318; E-mail: eaqliu@ntu.edu.sg.

\section{Introduction}

Vibroimpact is not an easily controlled phenomenon of structural dynamics. When resonance occurs, the system undergoes dangerously large oscillations. Resonance is generally to be avoided in designing structures, as the very large amplitude can cause structural failure, fatigue failure, discomfort, loud noises, and so on. Special vibroimpact damper or absorber can be introduced to reduce the effect resonance.

A bean bag damper is a very effective method to control the vibroimpact of a structural system. The conventional vibroimpact damper is essentially a rigid weight which moves freely between collisions with an excessively vibrating primary system. Its uni-directional movement is constrained by the ends of an attached on a confined space in the primary vibrating system. Attenuation of the primary system motion will occur, possibly by elasto-plastic contact deformations and certainly by momentum transfer if the collision is repeated and movements just before each collision are in opposite directions. When the primary system vibrates, the secondary mass moves back and forth colliding alternately on either end of the container. The amplitude of vibroimpact of the primary system is reduced significantly by the mechanism of transfer of momentum between primary and secondary system, and partly by the conversion of mechanical energy into wear and heat. A bean bag damper has a pot application to reduce the vibroimpact of antennas, gun mechanisms and flexible robot arms etc., and it is more appealing.

In the last three decades, vibroimpact dampers have undergone extensive research. Most studies have considered a single unit impact damper where the second mass was a solid body, such as Masri and Ibrahim [4] who studied the stochastic excitation of a single system. The coulomb friction of the damper was considered by Mansour and Filho [5] and Hunt and Crosslet [2] studied the coefficient of restitution in damping of vibroimpact. Lee and Byrun [3] gave a statistical analysis. The stability of periodic vibroimpact was investigated by Yasuda and Toyoda [10], Popplement et al. [7], and 
Bapat and Sankar [1], etc. Their work extended the knowledge on a rigid-body impact damper and led to successful application of the technology.

Usually, the single rigid body led to high magnitude of contact force and large noise. Consequently, the rigid second mass was replaced by a soft bag filled with balls of small diameter, and a resilient rather than a rigid impact damper was introduced. Semercigil and Popplew [9], Popplewell and Semercigil [8], and Pang et al. [6] pointed out that the bag ball system, often termed as a bean bag, to make a reduction in the peak force of the bean bag's resilience collision at the expense of elongation of the vibroimpact duration. According to this research work, it was found not only was a much quieter operation achieved, but the bean bag was more effective than the comparable rigid slug.

Despite utilization of several mathematical methods, such as linear momentum conservation on a single degree-of-freedom system, the theoretical simulation of a been bag vibroimpact damper has remained complex and great uncertainty in the predictions of the stiffness and damping factors remain because of a non-linear nature of system. A bean bag vibroimpact damper was grossly discontinuous, and therefore very difficult to analyse with procedures based upon continuum principles such as finite difference procedures. Although Discrete Element Methods could theoretically simulate complex systems with discontinuous materials such as a bean bag damper, it was important to know how to make a bean bag damper simple and effective in actual applications. Thus, there was a need to explore the dynamic performance of the vibroimpact damper to enable design optimisation.

The objective of this study was to establish a theoretical model from continuous mechanics point of view, and to describe overall performance of a bean bag rather than the movement of each individual ball. The bean bag was approximated as homogenous which enables the investigate of various critical parameters and the development of a general criteria for the design of effective vibroimpact dampers. In the experimental study, the bean bag damper was primarily designed for resonant attenuation. The natural frequency and mode shape of the primary system were measured. The optimised parameters of bean bag and the location of bean bag in the primary system for obtaining the best damper performance to control the vibroimpact were also determined.

\section{Mathematical model}

A vibroimpact damper is normally a small, rigid slug bag placed in a slightly larger cavity attached to a lightly damped resonant structure that is called the primary system. The bean bag is called the secondary system. The mathematical modelling combines the two systems in order to better understand and predict the bean bag dynamic performance in the whole system.

\subsection{Vibroimpact of a single-unit system}

A idealised vibroimpact system is shown in Fig. 1. Its primary system consisted of a linear spring with stiffness $K_{p}$, a viscous damping constant $C_{p}$, and mass $M_{p}$ excited by an external harmonic load $F \sin (\omega t)$. The secondary system was composed of a bean bag with an equivalent mass $M_{b}$, which could move uniaxially in a slot inside the usually much heavier mass $M_{p}$. The supposed frictionless motion of $M_{b}$ was instigated by collisions with $M_{p}$ which occur intermittently because of clearance $d / 2$. Figure 1 shown a forced single degree-of-freedom oscillatory system with motion limiting stops. Note that subscript $p$ and $b$ represent variables of the primary system and of the bean bag, respectively. The governing equation of motion of mass $M_{p}$ between impacts was

$$
\begin{aligned}
& \left(M_{p} \ddot{x}+C_{p} \dot{x}+K_{p} x\right)+\left(M_{b} \ddot{x}+C_{b} \dot{x}\right) \\
= & f(t),
\end{aligned}
$$

where $C_{b}$ was the equivalent damping coefficient of the bean bag, $f(t)$ was a external exciting force on the primary system. It was assumed that the system was linear and that the excitation amplitude $F$ and excitation frequency $\omega$ were constants. If the external excitation was given by

$$
f(t)=F e^{i \omega t}
$$

substituting Eq. (2) into Eq. (1) would lead to:

$$
\left(M_{p}+M_{b}\right) \ddot{x}+\left(C_{p}+C_{b}\right) \dot{x}+K_{p} x=F e^{i \omega t},(3)
$$

The particular solution to the above equation was a steady-state oscillation of the same frequency $\omega$ as that of the excitation. Assume the particular solution to be of the form

$$
x=X e^{i \omega t-\phi} \text {. }
$$

where $X$ was the amplitude of oscillation and $\phi$ was the phase of displacement with respect to the exiting force, and was the damped system forced natural frequency.

Substituting Eq. (4) into Eq. (1) would yield 

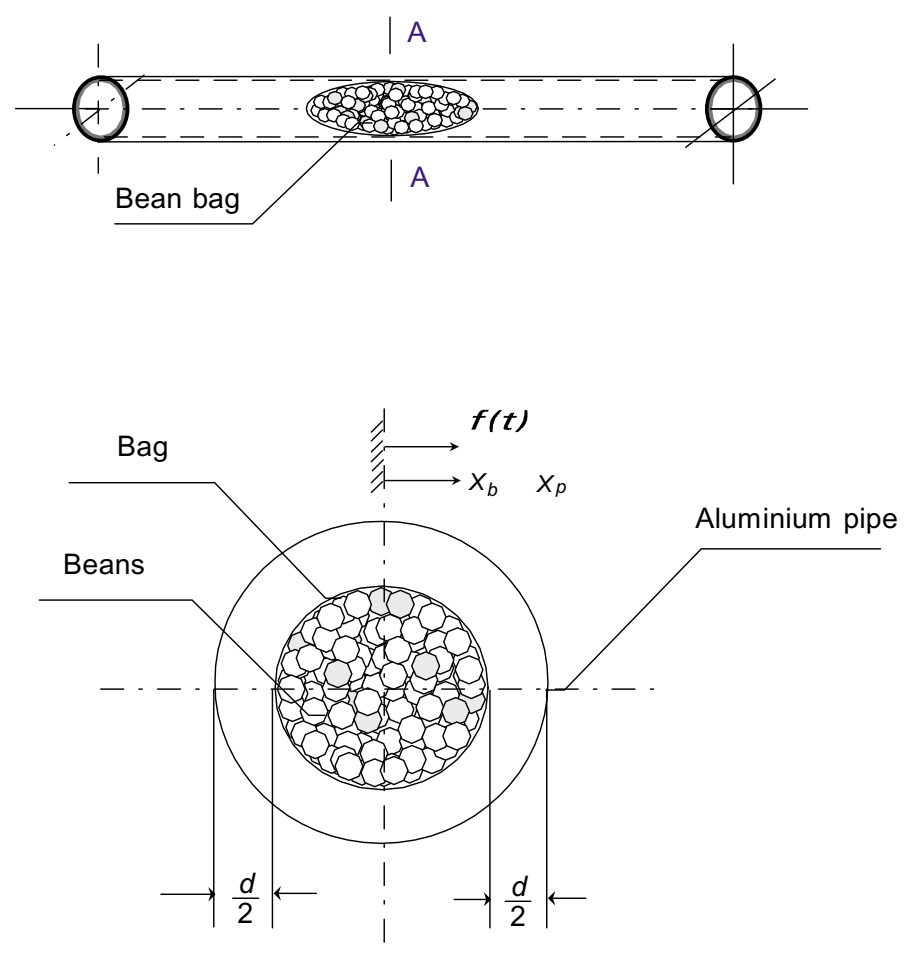

$\mathrm{A}-\mathrm{A}$

Fig. 1. Schematic diagram of bean bag vibroimpact systems.

$$
F(t)=\left[Z_{g}(\omega)+Z_{D}(X \omega)\right] \dot{X},
$$

where

$$
\begin{aligned}
& Z_{g}(\omega)=C_{p}+i\left(\omega M_{p}-\frac{K_{p}}{\omega}\right) ; \\
& Z_{D}(X, \omega)=C_{b}+i \omega M_{b} .
\end{aligned}
$$

The nondimensional expression for amplitude and displacement of Eq. (3) was

$$
\begin{aligned}
\frac{X_{s t}}{X}= & {\left[1-\left(\frac{\omega}{\omega_{n}}\right)^{2}\left(1+\mu \frac{M_{b}}{M_{p}}\right)\right] } \\
& +i\left[\mu\left(\frac{\omega}{\omega_{n}}\right)^{2} \frac{C_{b}}{M_{b} \omega}+\left(\frac{\omega}{\omega_{n}}\right)^{2} \frac{C_{p}}{M_{p} \omega}\right],
\end{aligned}
$$

where $X_{s t}$ was the static displacement of the system:

$$
X_{s t}=\frac{F}{K_{p}} ;
$$

$\mu$ was the ratio of mass:

$$
\mu=\frac{M_{b}}{M_{p}} ;
$$

$\omega_{n}$ was the natural frequency of the primary system:

$$
\omega_{n}=\sqrt{\frac{K_{p}}{M_{p}}} .
$$

Equation (7) would give the ratio between the static deformation and the maximum amplitude of the vibration of the primary system with a bean bag damper attached under the same magnitude of external force. Higher value of ratio indicated better performance of damper.

\subsection{Coefficient of restitution}

During impact, the relative motion of primary system and secondary system was often simply represented as half of a damped sine wave, according to the KelvinVoigt model. This was shown to be logically untenable, for it indicated that the bodies must exert tension on one another just before separating in simple onedimensional impact between two bodies in pure translation, the classical definition of coefficient of restitution e was the ratio of their relative speeds after impact to that before, so that 


$$
\dot{x}_{p}^{+}-\dot{x}_{b}^{+}=-e\left(\dot{x}_{p}^{-}-\dot{x}_{b}^{-}\right) .
$$

where $\dot{x}_{p}^{+}, \dot{x}_{p}^{-}$were impacting speed of primary system respectively before and after, and $\dot{x}_{b}^{+}, \dot{x}_{b}^{-}$were impacting speed of secondary system respectively before and after. Assuming that the motion of the bean bag was frictionless, speeds respectively before and after the impact were of the same magnitude but in opposite directions, i.e.

$$
\dot{x}_{p}^{+}=\dot{x}_{b}^{-} .
$$

Because of external excitation, the speeds of the primary system before and after impact were

$$
\dot{x}_{p}^{+}=-\omega X \sin \varphi ;
$$

and

$$
\dot{x}_{p}^{-}=-\omega X \sin (\omega t+\varphi) .
$$

where $\varphi$ was the phase of impact, i.e. when the impact starts. $x=X \cos \varphi$ and $X \cos (\varphi+\pi)$.

From Eqs. (9) to (11b), we could have

$$
\dot{x}_{b}^{+}(\tau)=-\omega X\left(\frac{1+e}{1-e}\right) \sin \varphi ;
$$

and

$$
\dot{x}_{b}^{-}\left(\frac{\pi}{\omega}+\tau\right)=-\omega X\left(\frac{1+e}{1-e}\right) \sin (\varphi+\pi) \cdot(
$$

where $\tau$ was reaction time between primary and secondary system.

\subsection{Energy loss}

In order to estimate the energy loss during impact, two variables, $W$ was the work done by impact force per cycle and $P$ was the rate of work in each cycle as follows:

$$
W=\int_{0}^{\frac{2 \pi}{\omega}} f(t) X d X
$$

and

$$
P=\int_{0}^{\frac{2 \pi}{\omega}} f(t) \dot{X} d X,
$$

where the impact force was

$$
f(t)=M_{b} \ddot{X}_{b}+C_{b} \dot{X}_{b} .
$$

Substitute Eq. (14) into Eq. (13) would lead to

$$
\begin{aligned}
W & =\int_{0}^{\frac{2 \pi}{\omega}}\left(M_{b} \ddot{X}_{b}+C_{b} \dot{X}_{b}\right) X d X \\
& =\pi C_{b} \omega^{2} X_{o}^{2} ;
\end{aligned}
$$

and

$$
\begin{aligned}
P & =\int_{0}^{\frac{2 \pi}{\omega}}\left(M_{b} \ddot{X}_{b}+C_{b} \dot{X}\right) \dot{X} d X \\
& =\pi M_{b} \omega^{3} X_{o}^{2}
\end{aligned}
$$

According to the principle of impulse and momentum, the following relationship held

$$
\int_{0}^{\tau} f(t) d t=M\left(\dot{x}_{b}^{+}-x_{b}^{-}\right)=-2 M_{b} \dot{x}_{b}^{+},
$$

and the average impact force within a cycle could be written as

$$
f(t)=-2 M_{b} \dot{x}_{b}^{+} / \tau .
$$

The impact force was zero between the two impacts during each cycle, thus the energy equations became

$$
W=4 M_{p} \omega X_{o}^{2}\left(\frac{1+e}{1-e}\right) \sin ^{2} \varphi ;
$$

and

$$
P=4 M_{p} \omega X_{o}^{2}\left(\frac{1+e}{1-e}\right) \cos \varphi \sin \varphi .
$$

Combining Eq. (15) with Eq. (18), we would have

$$
\frac{C_{b}}{M_{b} \omega}=\frac{4}{\pi}\left(\frac{1+e}{1-e}\right) \sin ^{2} \varphi ;
$$

and

$$
\frac{M_{b}}{M_{p}}=\frac{4}{\pi}\left(\frac{1+e}{1-e}\right) \sin \varphi \cos \varphi .
$$

The ratio $\frac{C_{b}}{M_{b} \omega}$ was the non-dimensional damping coefficient of bean bag. A higher value indicated a better performance. Substituting Eq. (19) into Eq. (6) would lead to

$$
\begin{aligned}
& \frac{X_{s t}}{X}= \\
& \left\{1-\left(\frac{\omega}{\omega_{n}}\right)^{2}\left[1+\frac{4 \mu}{\pi}\left(\frac{1+e}{1-e}\right) \sin \varphi \cos \varphi\right]\right\} \\
& +i\left\{\mu\left(\frac{\omega}{\omega_{n}}\right)^{2} \frac{4}{\pi}\left(\frac{1+e}{1-e}\right)^{2} \sin ^{2} \varphi\right. \\
& \left.+\left(\frac{\omega}{\omega_{n}}\right)^{2} \frac{C_{p}}{M_{p} \omega}\right\} .
\end{aligned}
$$

Note that the higher the ratio in Eq. (20), the better the damping effect. It was obvious from the above expression that one would prefer a higher damping coefficient $e$. 


\subsection{Clearance and mass ratio}

It was clear from Eq. (20) that the performance $\frac{X_{s t}}{X}$ of a bean bag system was mainly dependent on the frequency ratio $\lambda=\frac{\omega}{\omega_{n}}$, the mass ratio $\mu=\frac{M_{b}}{M_{p}}$, and the coefficient of restitution $e$. While under a stable motion, the clearance $d$ between bean bag and cavity could be defined as

$$
d=\left|\int_{0}^{\frac{\pi}{\omega}}\left[X_{b}^{+}-x(t)\right] d t\right|
$$

then

$$
\frac{d}{X}=\left|\pi\left(\frac{1+e}{1-e}\right) \sin \varphi-2 \cos \varphi\right| .
$$

where $e$ was the coefficient of restitution. The optimal points were $\frac{C_{b}}{M_{b} \omega}$ and $\frac{M_{b}}{M_{p}}$, depending on the value of $\frac{d}{X}$ for every $e$. Differentiating Eq. (22) with respect to $\varphi$ would give the optimisation of $\frac{d}{X}, \frac{C_{b}}{M_{b} \omega}$ and $\frac{M_{b}}{M_{p}}$ at $\varphi=\frac{\pi}{2}$

$$
\begin{aligned}
& \left.\frac{d\left(\frac{d}{X}\right)}{d \varphi}\right|_{\varphi=\frac{\pi}{2}}=\pi\left(\frac{1+e}{1-e}\right) ; \\
& \left.\frac{\left(\frac{C_{b}}{M_{b} \omega}\right)}{d \varphi}\right|_{\varphi \frac{\pi}{2}}=\frac{4}{\pi}\left(\frac{1+e}{1-e}\right) ; \\
& \left.\frac{d\left(\frac{M_{b}}{M_{p}}\right)}{d \varphi}\right|_{\varphi=\frac{\pi}{2}}=0 .
\end{aligned}
$$

When Eq. (20) was differentiated with respect to $\frac{\omega}{\omega_{n}}$, it would give

$$
\frac{\omega}{\omega_{n}}=\frac{1}{\sqrt{1+\mu \frac{M_{b}}{M_{p}}+\frac{C_{b}+C_{p}}{m_{p} \omega}}},
$$

then, the maximum of $\left|\frac{X}{X_{s t}}\right|$ could be written as

$$
\left|\frac{X}{X_{s t}}\right|_{\max }=\frac{1+\mu \frac{M_{b}}{M_{p}}+\frac{C_{b}+C_{p}}{M_{p} \omega}}{\sqrt{2} \mu+\frac{C_{b}+C_{p}}{M_{p} \omega}},
$$

If the system damping was very weak, i.e., $C_{p} \approx 0$, Eq. (25) became

$$
\left|\frac{X}{X_{s t}}\right|_{\max }=\frac{(1-e)}{4 \sqrt{2} \mu(1+e)}+\frac{1}{\sqrt{2}} .
$$

It was shown that with an increasing mass ratio, the value of $\left.\frac{X}{X_{s t}}\right|_{\max }$ reduced; but the reduction was only obvious at small mass ratios. According to the figure, at $\mu=0.03$, the bean bag system provided the most effective damping of the system vibration. When the ratio was bigger than 0.1 , the reduction was not effective. Thus a heavy bean bag damper, only increased the total weight of the system and probably the size of the structure without contributes much to the original purpose. Usually, for engineering practice, values of $\mu$ between 0.03 and 0.1 would be chosen.

\section{Experimental analysis}

The above theoretical analysis gave some preliminary results for how to design a bean bag vibroimpact damper. In particular, several other key parameters were identified and their influence discussed by experimental method. Based on this, an experiment investigating a vibroimpact damper system under excitation was designed.

\subsection{Experimental set-up}

The primary system investigated was an aluminium pipe and the impact damper used was a plastic bag filled with hardened steel bearing balls. The plastic bags and bearing balls were chosen due to their easy availability and varieties. The bean bag consisted of bearing balls of $\phi 4 \mathrm{~mm}, \phi 8 \mathrm{~mm}$ and $\phi 20 \mathrm{~mm}$, respectively, closely packed into a plastic bag with strings attached to both ends of the bag. The strings where to site the bean bag at the desired location within the pipe. The whole design of the bean bag and strings were considered as the secondary damper system. The aluminium pipe investigated was set up to simulate a free-free boundary condition; supported by strings hanging in the vertical plane while excitation was applied in the horizontal plane as shown in Fig. 2. This would enable us to study the damping effect on several fundamental frequencies at the same time.

Dimensions of the aluminium pipe were illustrated in Table 1. Prior to the commencement of test, the instrumentation system was calibrated whereby the pipe shown in Fig. 2 was replaced by a calibration mass of $1996 \mathrm{~g}$. Upon excitation, the signals detected by the force transducer and the accelerometer were analysed and a nearly constant magnitude was expected over the frequency range. Fine adjustment of the equipment sensitivity was made to achieve the desired magnitude. The system was then calibrated again at the end of all tests to ensure consistency in overall sensitivity of the system. 


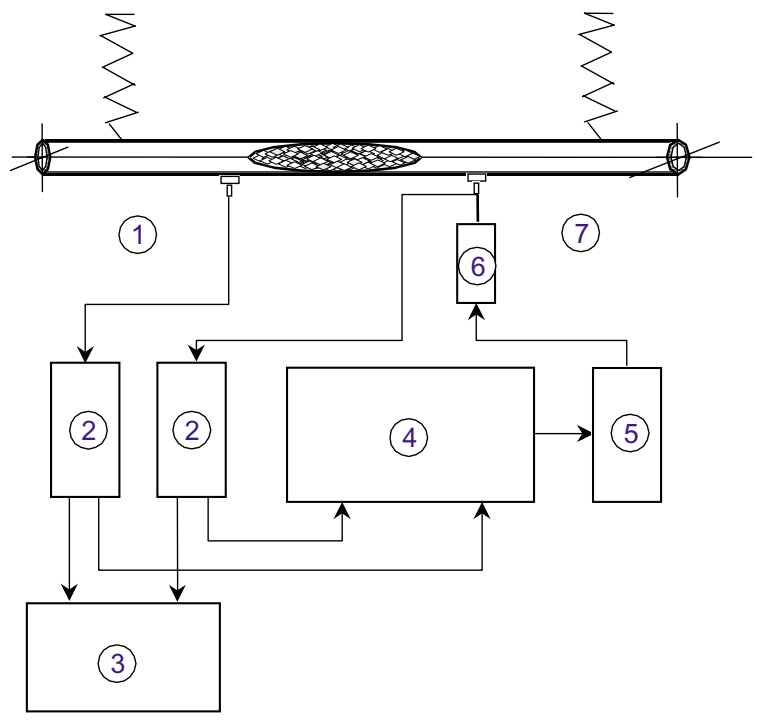

(1) Accelerometer, B \& K Type 4393

(2) Charge amplifier, B \& K Type 2635

(3) Digital storage oscilloscope, Tektronix 2211

(4) Multichannel analysis system, B \& K Type 3550

(5) Power amplifier, PA-118

(6) Electrodyamic shaker, ET-126

(7) Force transducer, B \& K Type 8200

Fig. 2. Schematic diagram of experimental set-up for bean bag vibroimpact system.

Table 1

Parameters of primary system

\begin{tabular}{cc}
\hline Aluminium pipe & Parameters \\
\hline Mass & $0.332 \mathrm{~kg}$ \\
Length & $1.39 \mathrm{~m}$ \\
External diameter & $26.0 \mathrm{~mm}$ \\
Internal diameter & $23.5 \mathrm{~mm}$ \\
Young' modulus & $69 \mathrm{GPa}$ \\
\hline
\end{tabular}

\subsection{Experimental procedure}

The following set of procedure was carried out for all tests performed. Firstly, the equipment as shown in Fig. 2 was set up. Secondly, with the signal generator (built-in B\&K mullet-channel analysis system, Type 3550) activated, the gain of power amplifier was adjusted to an indicated level (marked out by the experimenter). Next, the system was left to run for 10 minutes before any measurements were taken, allowing the system to stabilise. Finally, three consecutive sets of frequency responses were then obtained for each particular configuration.

A reference frequency response (natural response of primary system without damper) was obtained prior to the commencement of the experiments conducted for every different set of tests. Not all influential parameters identified in the theoretical analysis can be systematically tested, for example, the coefficient of restitution $e$. It was impossible to control precisely the value of e since during the collisions in the cavity, the tightness of the bean bag would change, which would eventually lead to a change in $e$. Due to the small bore of aluminium pipe used in this test, it was also impossible to test different values of the tightness of the bag. But as noted by Pang et al. [6] from their tests, the attenuation of vibration was virtually unaffected by the tightness of bean bag packaging. Thus, only three sets of tests were conducted for the following parameters: mass ratio, size of balls and the position of bean bag in the pipe.

\subsection{Size of bearing balls, mass ratio and position of damper}

The mass ratio was varied by changing the number of bearing balls $(\phi 4 \mathrm{~mm})$ packed in the bean bags. The ratio was varied from 5\% to $14 \%$ at $1 \%$ increment. In the tests, the bean bag was placed at the mid span of pipe as the primary concern was to reduce the amplitude of the first mode vibration. Then fixed at a mass ratio of $10 \%$, bean bags with bearing balls of different sizes ( $\phi 4 \mathrm{~mm}, \phi 8 \mathrm{~mm}, \phi 10 \mathrm{~mm}$ and $\phi 20 \mathrm{~mm})$ were studied. The bean bag was positioned at various locations along the pipe and at the mid span of pipe. The aluminium pipe natural frequencies of the first three modes were given in Fig. 3. The phenomena of "twin peaks" were observed for the third mode. The presence of twin peaks was due to the slight non-symmetry in cross-section of pipe resulting in the exhibition of two close modes. On the whole, the natural frequencies determined experimentally were relatively close to the calculated values.

The magnitude at the second mode $(220 \mathrm{~Hz}-235 \mathrm{~Hz})$, however, was relatively low. This was due to the sensitivity of frequency response analysis to the location of response point. As the accelerometer was positioned at or very close to the node of the structure's second mode, it was very difficult to make effective measurement of this particular mode. Hence, for the evaluation of the bean bag effectiveness, only the first and third modes were considered; with the first mode attenuation being 


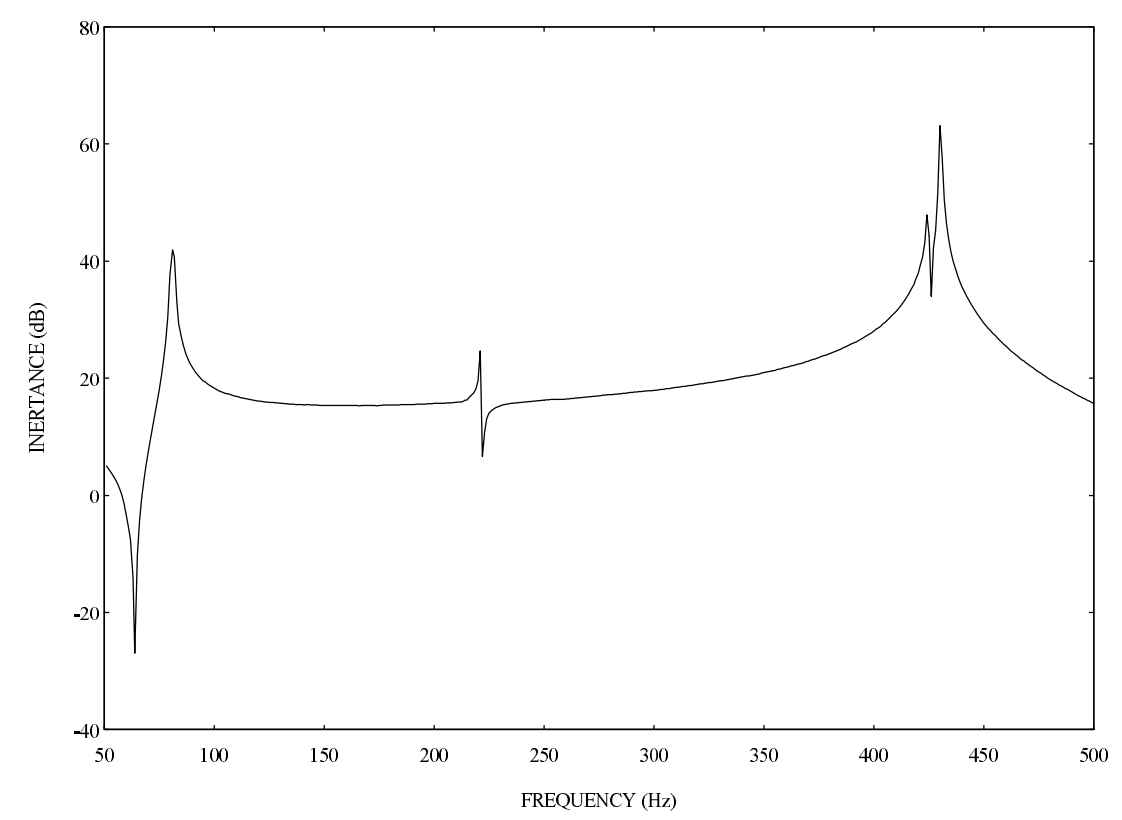

Fig. 3. Frequency response of primary system.

the primary concern. Figures 4 and 5 shown the percentage reduction in vibration amplitude with respect to variations in the size of bearing balls and mass ratio. Note that the bean bag was placed at the mid span of pipe during these tests.

For different sizes of ball bearings as shown in Fig. 4, where the mass ratio was kept at $8 \%$, the percentage of reduction increases with a smaller diameter of balls. This was because smaller balls provide more contact surface thus dissipate more energy. However this did not mean that balls should be as small as possible. When the particles in the bag were too fine, the behaviour would be more fluid-like and energy dissipation due to internal contact motion would be reduced. Furthermore, it appeared that the third mode attenuation was found to be generally better than the first mode and not sensitive to the ball diameter.

As for the variation of mass ratio, Figure 5 shown that for balls of $4 \mathrm{~mm}$, at a mass ratio of $8 \%$, the percentage of reduction for modes 1 and 3 reaches more than $90 \%$. Further increase in mass ratio did not significantly improve the performance. Figure 6 shown that the attenuation was greater when the bean bag was positioned at or around the antinodes of the particular modes. For the third mode vibration, the attenuation was observed to be relatively more significant than the first mode.

All experimental results were tabulated in Tables 24. The performance of bean bag was encouraging and the experimental results would be discussed below.

\subsection{Discussion of experimental results}

In the study of performance of a bean bag damper, the mass of primary system, an aluminium pipe, was merely $332 \mathrm{~g}$. To ensure minimum alterations to the characteristics of the primary system, the mass of damper used was limited to a mass ratio of $14 \%$. This was important as the introduction of additional mass would shift the natural frequencies of primary system and might not be desired in some applications. In addition, the fundamental mode attenuation was of prime concern. The first parameter being investigated was the variation of size of bearing balls. This was done to select an optimum diameter of the ball. As depicted by Fig. 4 , the diameter of $4 \mathrm{~mm}$ ball bearings exhibited superior damping efficiency. It was expected that smaller balls would give a better damping performance. This could not be tested, normally, due to unavailability of the balls. An optimal ball size should exist which would give the best damping effectiveness.

With the selection of $4 \mathrm{~mm}$ diameter steel balls, the variation in mass ratio was studied. As seen in Fig. 5, the reduction of amplitude increased with mass ratio for mode 1 and 3. At a mass ratio of $8 \%$, the amount of reduction reached an order of $90 \%$. With the selection of a mass ratio of $8 \%$, therefore, the vibration amplitude of the primary system was reduced by the maximum effectiveness of $90 \%$. Moreover, an additional mass added to the primary system would alter the characteristics of system to a greater extent. 


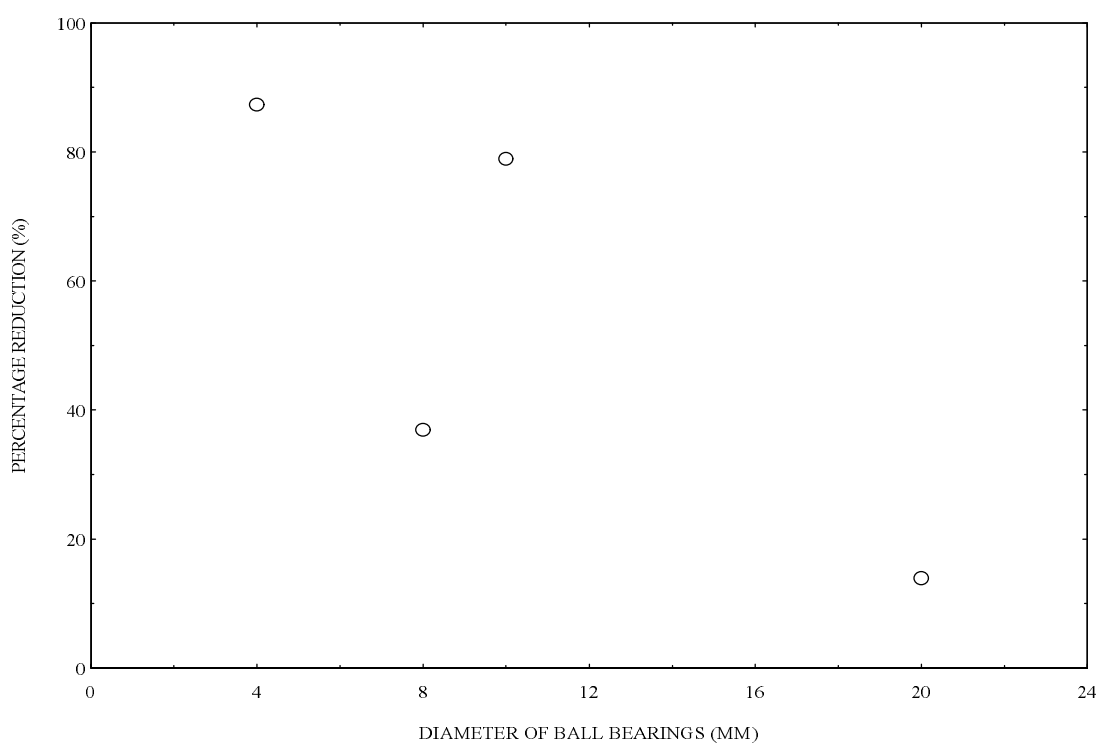

(a) First Mode Attenuation

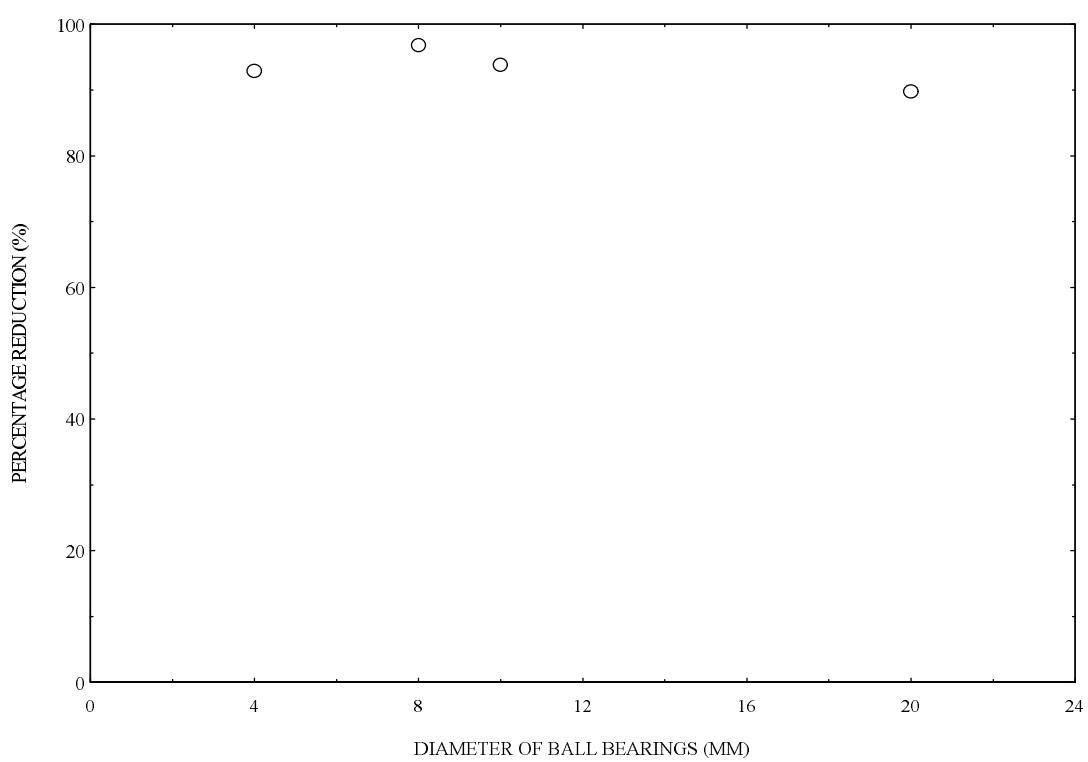

(b) Third Mode Attenuation

Fig. 4. Variation of vibration reduction with size of ball bearing.

At this juncture, it should be noted that the positions of excitation (from shaker) and signal reception (through accelerometer) contributed to the difference in the responses obtained for different modes. As the accelerometer was located at node of the second mode vibration, only the first and third modes were considered, with the former being the primary interest.

Finally, with the bean bag placed at different positions along the pipe, it could be observed that the attenuation of vibration was more pronounced at the antinodes of the respective mode shapes. This verified that the bean bag was a resonant damper and the position of bean bag would be critical for its respective applications.

\section{Conclusions}

In spite of superior damping performance of a bean bag damper, there were several limitations to the ap- 


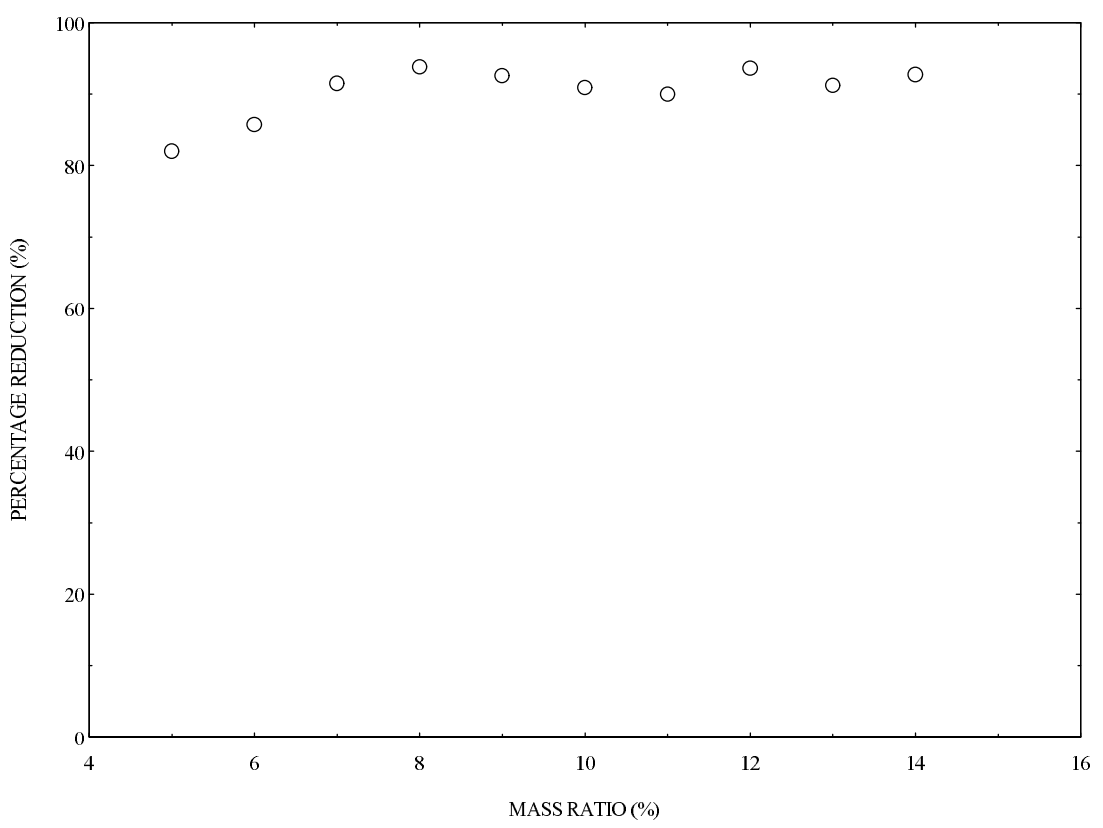

(a) First Mode Attenuation

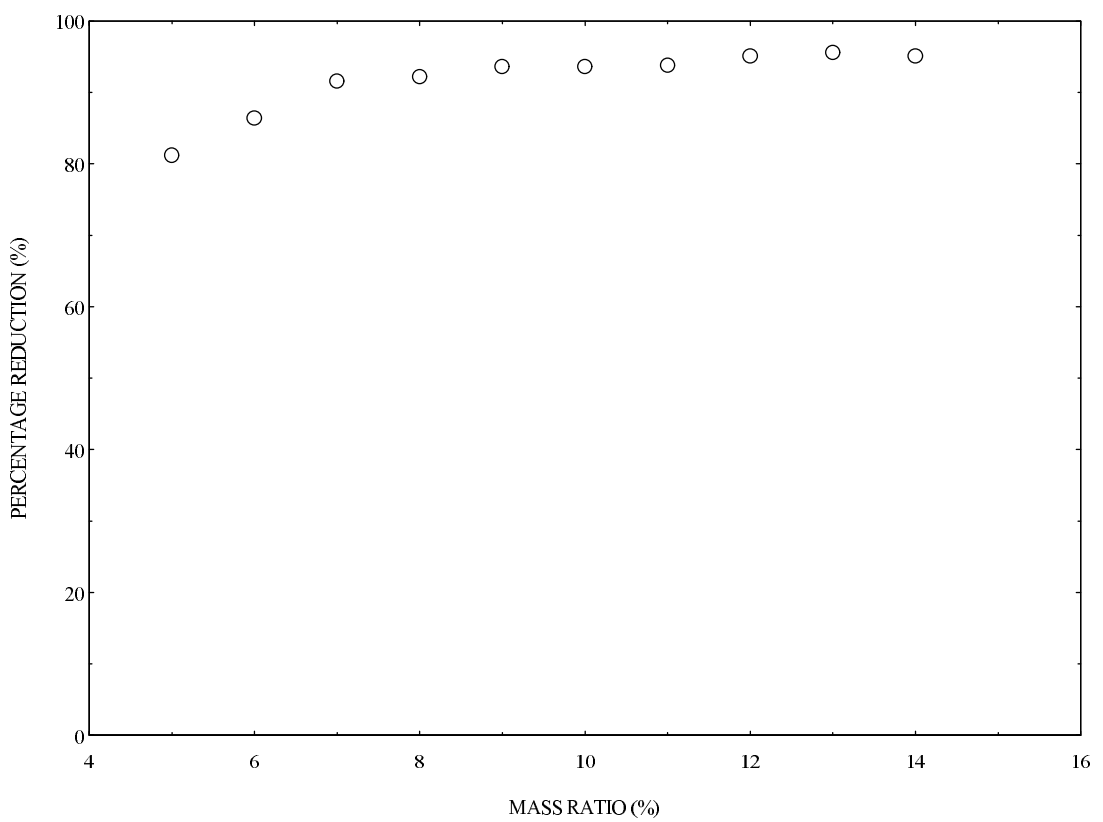

(b) Third Mode Attenuation

Fig. 5. Variation of vibration reduction with mass ratio.

plication of damper. Firstly, the design of a bean bag damper required consideration of the entire sophisticated system and its behavioural characteristics have yet been fully developed. Moreover, exact mathematical modelling of bean bag has not been fully established. Hence, the effectiveness of this type of damper has to be determined experimentally, and extra effort will be required for its application.

Secondly, a bean bag being a directional resonant damper might only be effective if the primary system has discrete resonant characteristics. In addition, systems subjected to random vibration in various direc- 


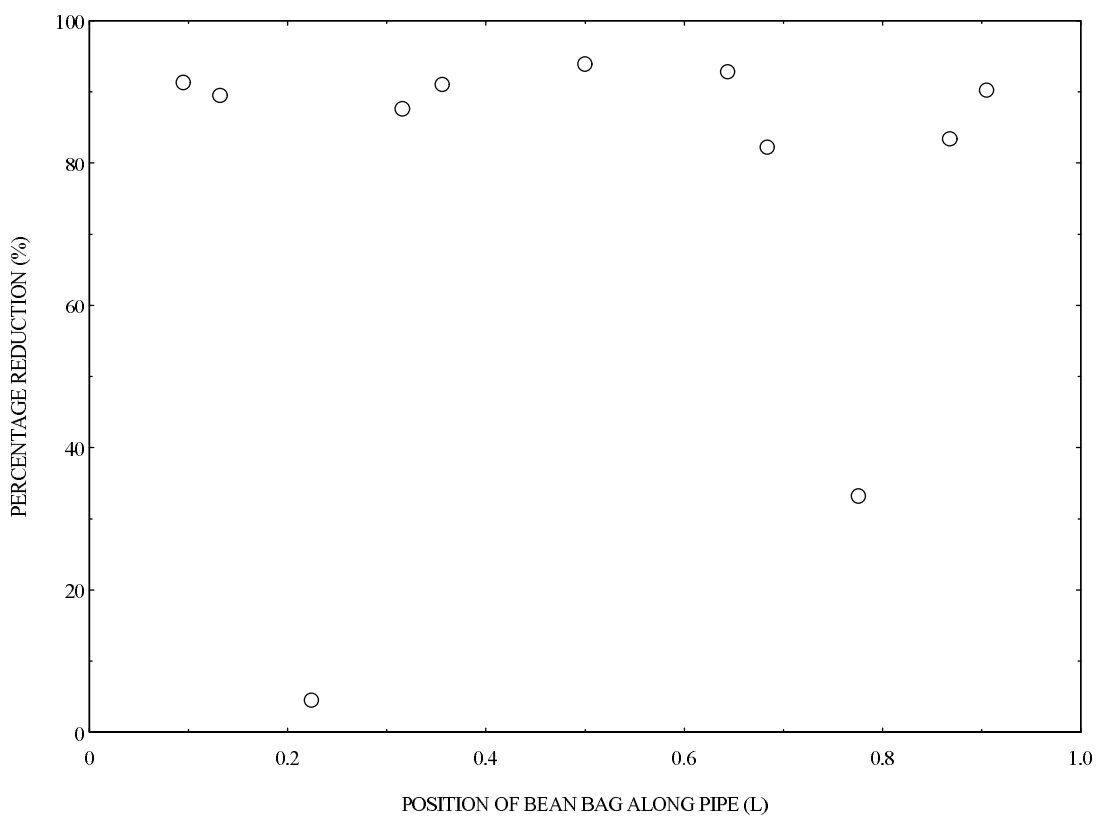

(a) First Mode Attenuation

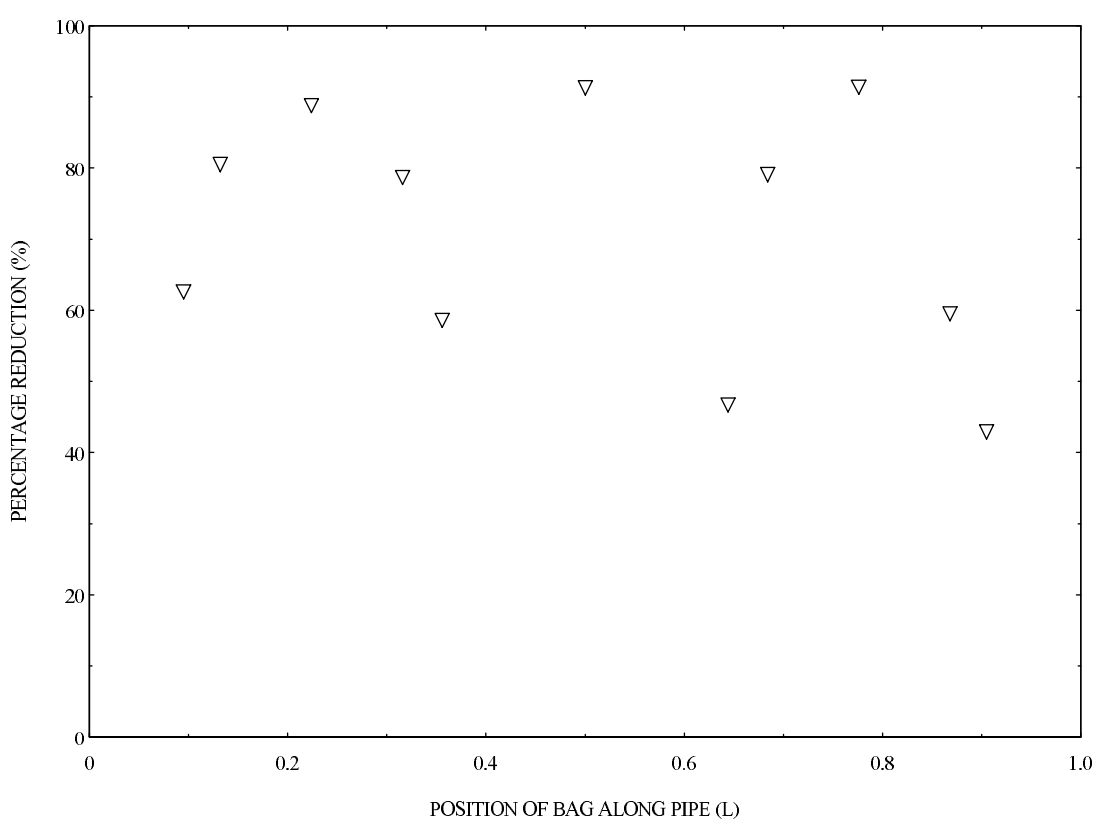

(b) Third Mode Attenuation

Fig. 6. Variation of vibration reduction with position of damper.

tions might not attain the desired level of attenuation as a bean bag damper is effective in one or two certain directions. Also, the installation of a bean bag damper posed another constraint to its application. As the location of damper relative to the primary system is critical for its damping performance, the availability of space to install at effective location would be important. Furthermore, the container which holds the bean bag, which is then mounted to the primary system has to be properly designed to ensure that the various criteria responsible for the damping performance of the bean bag damper are satisfied. Consolidating the results of 
Table 2

Variation of ball bearings size (mass ratio 8\%)

\begin{tabular}{|c|c|c|c|c|c|c|}
\hline \multirow{3}{*}{$\begin{array}{c}\text { Diameter of Ball } \\
\text { Bearings (mm) }\end{array}$} & \multicolumn{6}{|c|}{ Reduction in Vibration Amplitude } \\
\hline & \multicolumn{2}{|c|}{ Mode 1} & \multicolumn{2}{|c|}{ Mode 2} & \multicolumn{2}{|c|}{ Mode 3} \\
\hline & $\mathrm{dB}$ & $\%$ & $\mathrm{~dB}$ & $\%$ & $\mathrm{~dB}$ & $\%$ \\
\hline 4 & 17.9 & 87.3 & 3.1 & 30.0 & 23.0 & 92.9 \\
\hline 8 & 4.0 & 36.9 & -0.4 & -4.7 & 29.8 & 96.8 \\
\hline 10 & 13.5 & 78.9 & -1.6 & -20.2 & 24.1 & 93.8 \\
\hline 20 & 1.3 & 13.9 & 4.0 & 36.9 & 19.8 & 89.8 \\
\hline
\end{tabular}

Table 3

Variation of mass ratio (ball diameter $4 \mathrm{~mm}$ )

\begin{tabular}{|c|c|c|c|c|c|c|}
\hline \multirow[t]{3}{*}{ Mass Ratio (\%) } & \multicolumn{6}{|c|}{ Reduction in Vibration Amplitude } \\
\hline & \multicolumn{2}{|c|}{ Mode 1} & \multicolumn{2}{|c|}{ Mode 2} & \multicolumn{2}{|c|}{ Mode 3} \\
\hline & $\mathrm{dB}$ & $\%$ & $\mathrm{~dB}$ & $\%$ & $\mathrm{~dB}$ & $\%$ \\
\hline 5 & 14.9 & 82.0 & 0.9 & 9.8 & 14.5 & 81.2 \\
\hline 6 & 16.9 & 85.7 & 1.0 & 10.9 & 17.3 & 86.4 \\
\hline 7 & 21.4 & 91.5 & 1.6 & 16.8 & 21.5 & 91.6 \\
\hline 8 & 24.1 & 93.8 & 3.2 & 30.8 & 22.2 & 92.2 \\
\hline 9 & 22.6 & 92.6 & 1.6 & 16.8 & 23.9 & 93.6 \\
\hline 10 & 20.8 & 90.9 & 0.9 & 9.8 & 23.9 & 93.6 \\
\hline 11 & 20.0 & 90.0 & -1.2 & -14.8 & 24.1 & 93.8 \\
\hline 12 & 23.9 & 93.6 & -2.6 & -34.9 & 26.2 & 95.1 \\
\hline 13 & 21.1 & 91.2 & -1.1 & -13.5 & 27.1 & 95.6 \\
\hline 14 & 22.7 & 92.7 & -0.4 & -4.7 & 26.2 & 95.1 \\
\hline
\end{tabular}

Table 4

Variation of position of damper (mass ratio $8 \%$ and ball diameter $4 \mathrm{~mm}$ )

\begin{tabular}{|c|c|c|c|c|c|c|}
\hline \multirow[t]{3}{*}{ Position of damper (x L) } & \multicolumn{6}{|c|}{ Reduction in Vibration Amplitude } \\
\hline & \multicolumn{2}{|c|}{ Mode 1} & \multicolumn{2}{|c|}{ Mode 2} & \multicolumn{2}{|c|}{ Mode 3} \\
\hline & $\mathrm{dB}$ & $\%$ & $\mathrm{~dB}$ & $\%$ & $\mathrm{~dB}$ & $\%$ \\
\hline 0.095 & 21.2 & 91.3 & 3.5 & 33.2 & 8.6 & 62.8 \\
\hline 0.132 & 19.6 & 89.5 & 5.8 & 48.7 & 14.3 & 80.7 \\
\hline 0.224 & 0.4 & 4.5 & 1.5 & 15.9 & 19.2 & 89.0 \\
\hline 0.316 & 18.1 & 87.6 & 1.2 & 12.9 & 13.5 & 78.9 \\
\hline 0.356 & 20.9 & 91.0 & 0.8 & 8.8 & 7.7 & 58.8 \\
\hline 0.5 & 24.3 & 93.9 & -0.7 & -8.4 & 21.4 & 91.5 \\
\hline 0.644 & 22.8 & 92.8 & 5.4 & 46.3 & 5.5 & 46.9 \\
\hline 0.684 & 15.0 & 82.2 & 2.2 & 22.4 & 13.7 & 79.3 \\
\hline 0.776 & 3.5 & 33.2 & 4.8 & 42.5 & 21.5 & 91.6 \\
\hline 0.868 & 15.6 & 83.4 & -0.6 & -7.2 & 7.9 & 59.7 \\
\hline 0.905 & 20.2 & 90.2 & 3.6 & 33.9 & 4.9 & 43.1 \\
\hline
\end{tabular}

various analyses performed in this project, the following procedure may be adopted in designing an impact damper, particularl a been bag damper:

(a) determine the inherent characteristics and natural responses of the structure of interest;

(b) identify the type of loading(s) that would be experienced by the structure;

(c) locate the appropriate site for impact damper with respect to the type of loading (excitation) and space availability;

(d) choose the optimum mass ratio and clearance distance (if possible) for the structure.
The above procedure was derived based on the specific configuration of the bean bag damper studied, and thus might not be applicable to dampers of other configurations. However, the trends observed should generally be valid and further justifications would definitely be favourable.

\section{Acknowledgement}

The authors acknowledge the Defence Technology Group for funding the project under grant NTU/MINDEF 11/94. 


\section{References}

[1] C.N. Bapat and S. Sankap, Single unit impact damper in free and forced vibration, Journal of Sound and Vibration 1 (1985), 85-94.

[2] K.H. Hunt and F.R.E. Crossley, Coefficient of restitution interpreted as damping in vibroimpact, Journal of Applied Mechanics 6 (1975), 440-445.

[3] C.E. Lee and K.P. Byrne, Impact statistics for a simple random rattling system, Journal of Sound and Vibration 3 (1987), 529-543.

[4] S.F. Masri and A.M. Ibrahim, Stochastic excitation of a simple system with impact damper, Earthquake Engineering and Structural Dynamics 1 (1973), 337-346.

[5] W.M. Mansour and D.R.T. Filho, Impact dampers with coulomb friction, Journal of Sound and Vibration 3 (1974),
247-265.

[6] C. Pang, N. Popplewell and S.E. Semercigil, An overview of a bean bag damper's effectiveness, Journal of Sound and Vibration 2 (1989), 359-363.

[7] N. Popplewell, C.N. Bapat and K. McLachlan, Stable periodic vibroimpact of an oscillator, Journal of Sound and Vibration 1 (1983), 41-59.

[8] N. Popplewell and S.E. Semercigil, Performance of the bean bag impact damper for a sinusoidal external force, Journal of Sound and Vibration 2 (1989), 193-223.

[9] S.E. Semercigil and N. Popplewell, The bean bag impact damper, Proceeding of the Third International Conference on Recent Advances in Structure Dynamics 1 (1988), 459-468.

[10] K. Yasuda and M. Toyoda, The damping effect of an impact damper, Bulletin of the JSME 153 (1978), 621-752. 

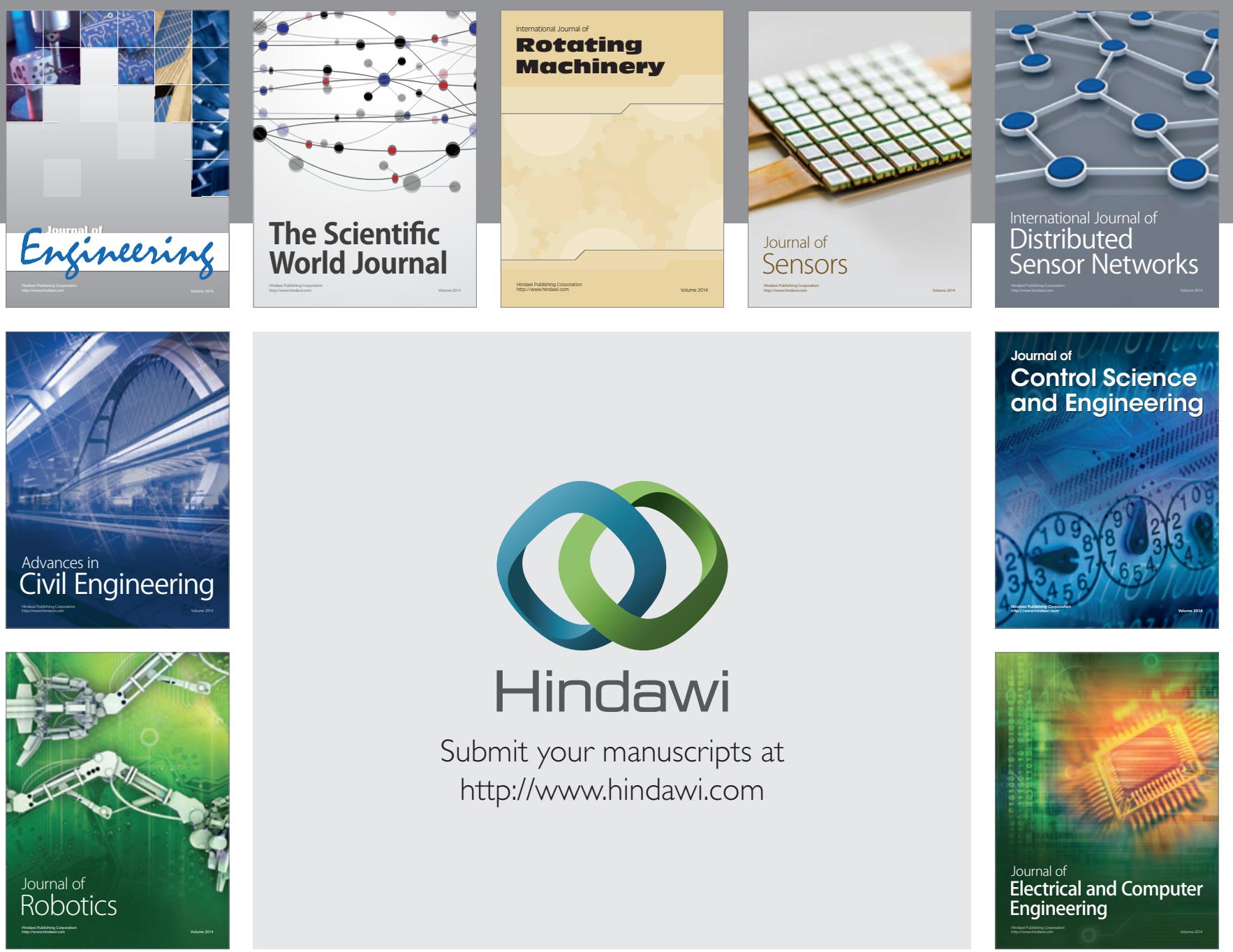

Submit your manuscripts at

http://www.hindawi.com
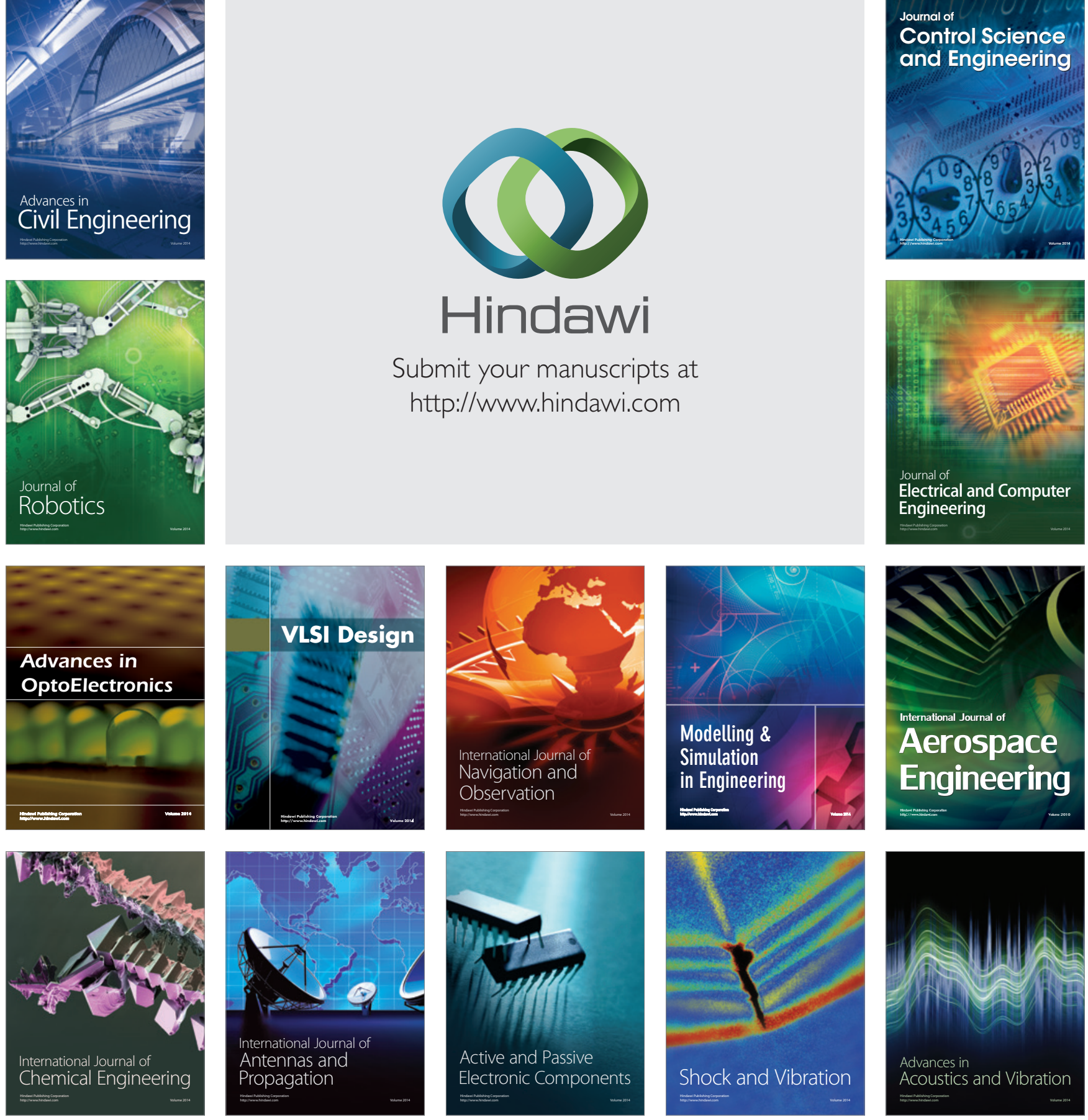